\title{
Absolutely relative or relatively absolute: violations of value invariance in human decision making
}

\author{
Andrei R. Teodorescu ${ }^{1} \cdot$ Rani Moran ${ }^{2} \cdot$ Marius Usher $^{2}$
}

Published online: 29 May 2015

(C) Psychonomic Society, Inc. 2015

\begin{abstract}
Making decisions based on relative rather than absolute information processing is tied to choice optimality via the accumulation of evidence differences and to canonical neural processing via accumulation of evidence ratios. These theoretical frameworks predict invariance of decision latencies to absolute intensities that maintain differences and ratios, respectively. While information about the absolute values of the choice alternatives is not necessary for choosing the best alternative, it may nevertheless hold valuable information about the context of the decision. To test the sensitivity of human decision making to absolute values, we manipulated the intensities of brightness stimuli pairs while preserving either their differences or their ratios. Although asked to choose the brighter alternative relative to the other, participants responded faster to higher absolute values. Thus, our results provide empirical evidence for human sensitivity to task irrelevant absolute values indicating a hard-wired mechanism that precedes executive control. Computational investigations of several modelling architectures reveal two alternative accounts for this phenomenon, which combine absolute and relative processing. One account involves accumulation of differences with activation dependent processing noise and the other emerges from accumulation of absolute values
\end{abstract}

Electronic supplementary material The online version of this article (doi:10.3758/s13423-015-0858-8) contains supplementary material, which is available to authorized users.

Andrei R. Teodorescu ateodore@indiana.edu

1 Psychological and Brain Science, Indiana University, 1101 E. 10th Street, Bloomington, IN 47405, USA

2 Tel-Aviv University, Tel Aviv, Israel subject to the temporal dynamics of lateral inhibition. The potential adaptive role of such choice mechanisms is discussed.

Keywords Computational modeling · Judgment and decision making $\cdot$ Response time models $\cdot$ Inhibition

\section{Introduction}

A central goal of the cognitive, economic, and biological sciences has been to uncover the mechanism underlying human and animal decision-making behavior. Decisions are made based on a set of values that characterizes the choice alternatives (e.g., monetary rewards, likelihoods of events, perceptual properties or subjective utilities). However, when aiming to select the best out of a given set of alternatives, processing information about the absolute value of each alternative can be considered superfluous. Indeed, leading theories of human decision making postulate that task irrelevant information about absolute values is discarded in favor of relative value representations. This relativity of information processing in decision making is crucial for producing context effects and has been formulated both in terms of differences between values (Ratcliff and Rouder 1998; Roe et al. 2001; Tversky and Simonson 1993; Usher and McClelland 2004) and value ratios (Brown and Heathcote 2008; Louie et al. 2013). Concurrently, other theories used to describe decision making in both humans (Usher and McClelland 2001) and decentralized biological systems (e.g., bee colonies; Pais et al. 2013) suggest that some sensitivity to absolute values is, in fact, retained. Value sensitive mechanisms have also been argued to exhibit adaptive advantages, such as breaking decision deadlock between equally valued alternatives faster for high value alternatives than for low value alternatives 
(Pirrone et al. 2014). Two questions are thus begged in this study: (a) Does human decision making retain sensitivity to absolute information values, above and beyond their relative properties? and if so (2) by means of what underlying mechanism do absolute value sensitivity and relative value representation coexist?

Ample evidence from psychology and biology indicates that choices and their latencies are the result of a process that sequentially accumulates samples of noisy, momentary, evidence values towards an internal decision criterion (Marshall et al. 2009; Meyer et al. 1988; Ossmy et al. 2013; Ratcliff and Rouder 1998; Ratcliff 1978; Teodorescu and Usher 2013; Usher and McClelland 2004). In this sequential sampling framework, a relative model is one that either accumulates relative momentary values (e.g., momentary evidence value differences) or, alternatively, accumulates absolute values but implements a relative stopping rule (e.g. stop when the difference between accumulators crosses a criterion amount). While values often are associated with economic rewards, perceptual values also constitute an informational basis for decision making and perceptual decision-making manifests many biases and context effects associated with economic decision making (Trueblood et al. 2013; Tsetsos et al. 2012). For binary decisions, accumulation of value differences as in the Drift Diffusion Model (DDM; Ratcliff 1978), has been related to choice optimality, as a mechanistic implementation of the Bayesian Sequential Probability Ratio Test (Wald and Wolfowitz 1948 but see Drugowitsch et al. 2012; Moran 2014). The DDM has been successful in fitting a plethora of experimental results and has been suggested as a unifying framework for both perceptual and economic decision making (Basten et al. 2010; Ratcliff and McKoon 2008; Towal et al. 2013). Similarly, fractional normalization — an algorithm that divides each individual input value by the sum of all input values, ${ }^{1}$ thus discarding absolute values in favor of ratioshas been recently proposed as a canonical neural computation that permeates all levels of brain functions (Carandini and Heeger 2012; Louie et al. 2011; Louie et al. 2013). In this study, we focused on these two relative processes.

When considering the type and degree of information processing relativity in different model architectures, distinct dependencies between absolute model inputs and model outputs become readily evident. Specifically, both pure difference (pure DDM) and ratio (pure normalization) relations are

\footnotetext{
${ }^{1}$ Note that fractional normalization is a specific case of normalization that is purely relative. Mechanistically, this is achieved through inputs inhibiting each other so that only momentary ratios are accumulated. Normalization in its general definition, however, also includes a saturation parameter that allows an adaptive, continuous transition between purely relative, fractional normalization, and asymptotically absolute values. This issue will be addressed more thoroughly in the Methods and Computational sections.
}

invariant to manipulations that maintain the difference or the ratio of absolute input values, respectively. In this study, we use a binary perceptual decision paradigm, requiring decision makers to choose the brighter out of a pair of fluctuating gray patches (i.e., evaluate relations between brightness stimuli while ignoring absolute values). Brightness value manipulations were specifically designed to test different types of relativity in the processing of decision information. Despite the relative nature of the task, we present results demonstrating surprising sensitivity of human choices and choice-latencies to task irrelevant absolute information values. An extensive computational investigation of corresponding accumulation models demonstrates that the observed sensitivity to absolute values conforms to neither pure difference nor ratio relations. Begging a principled synthesis, we put forth the proposition that the observed sensitivity to absolute values can be captured by two plausible, although as we will show, apparently incompatible, origins: (a) the dependence of processing noise on input intensity (Brunton et al. 2013; Lu and Dosher 2008) when implemented within a DDM framework or (b) response competition produced by lateral inhibition between evidence accumulators and the resulting temporal dynamics (Teodorescu and Usher 2013; Usher and McClelland 2001, 2004).

\section{Experimental methods}

To test the invariance predictions of purely relative information processing assumptions, we manipulated, in two experiments, the mean overall brightness intensity of two simultaneously presented, spatially separated, temporally fluctuating, gray patches (Fig. 1a and b). Critically, compared to a baseline condition (upper panel; Fig. 1b), the manipulation maintained constant either the difference of the means (additive-boost condition; middle panel; Fig. 1b) or their ratio (multiplicative-boost condition; lower panel; Fig. 1b). All conditions were randomly intermixed and on each trial, subjects were asked to choose the brighter patch as fast and as accurately as possible but were not given any information concerning the existence of different intensity conditions. To control for the differences in mean stimulus onset brightness between the experimental conditions, in Exp. 2 all trials initiated with a matching $100 \mathrm{~ms}$ (6 frames) baseline period after which, depending on condition, mean brightness values were identical to the ones in Exp. 1

\section{Participants}

Eight Tel-Aviv University undergraduate students participated in Exp. 1 and eight in Exp. 2, in exchange for course credit. Each participant was tested in two, 60-minute sessions (on different days but no more than 4 days apart). One subject in 


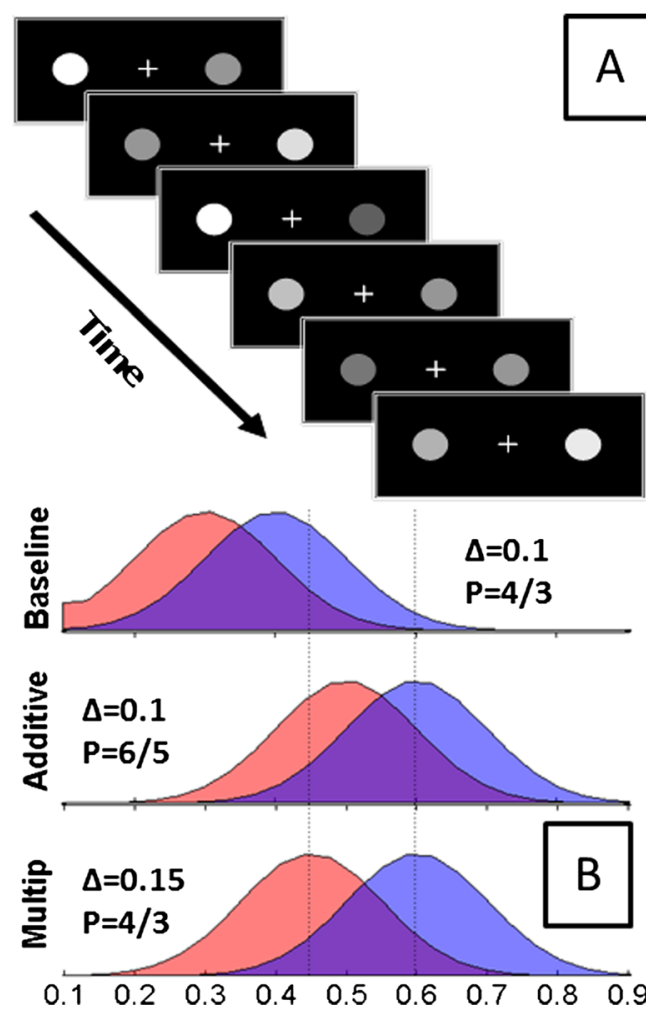

Fig. 1 Top left (a) time course of an experimental trial demonstrating a sequence of evidence samples presented (at a $60-\mathrm{Hz}$ refresh rate) until response. Bottom left (b) Brightness value distributions in each condition for the target (blue) and non-target (red) alternatives. In the baseline condition the means of the brightness distributions (normal with equal variance, top panel) were set to 0.3 and 0.4 (on a 0-black to 1 -white scale), for the nontarget and the target, respectively. The baseline mean brightness difference was $\Delta=0.1$ and the ratio of the means was $P=4 / 3$ (top panel). The baseline condition acted as a reference point for the other boost conditions. The brightness means for the additive-boost condition

Exp. 1 was excluded from the analyses due to experimenter error (the subject performed both sessions consecutively on the same day and consequently RTs were more than 3 standard deviations slower than the rest of the group). One subject in Exp. 2 did not complete the second session and was also excluded from the analyses. Exclusion of these subjects did not affect the general pattern of our results, and all reported data in the remainder of the paper are from the remaining seven participants. All participants had normal or corrected to normal vision. The projects were approved by the department's ethical committee.

\section{Materials}

All stimuli in this experiment were presented on a ViewSonic Graphics Series G90fB 19" CRT monitor. The monitor was gamma corrected so that physical brightness outputs (in lumens) are linear with respect to MATLAB RGB values where zero represents the minimum screen brightness and one

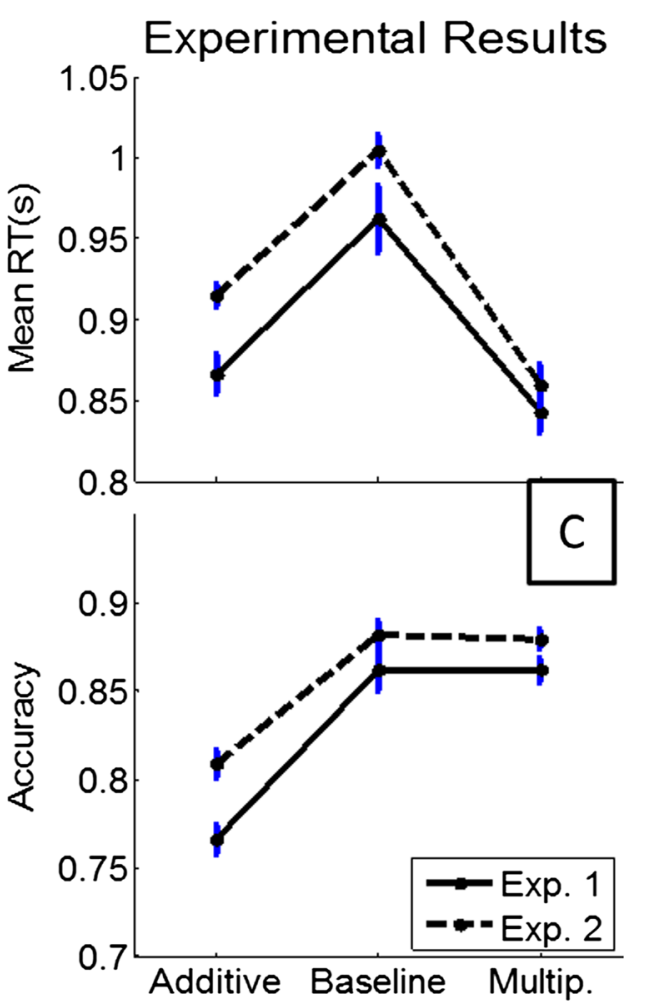

were generated by increasing both baseline distribution means by 0.2 $([0.4,0.3]+0.2=[0.6,0.5])$, thus preserving the $\Delta=0.1$ difference between these means while decreasing the ratio of means to $P=6 / 5$; middle panel). The multiplicative-boost brightness means are generated by multiplying both baseline mean brightness values by a factor of 1.5 ([0.4, $\left.0.3]^{*} 1.5=[0.6,0.45]\right)$ thus preserving their $P=4 / 3$ ratio while increasing the difference to $\Delta=0.15$ (bottom panel; see also Teodorescu and Usher (2013), Exp. 3). Right (c) experimental results: mean correct RT (top panel) and accuracy (bottom panel). Error bars represent within subject standard errors (Cousineau 2005), within each experiment

represents the maximum screen brightness. Note that the range of available screen brightness is only a small section of the full brightness range from no light up to some extremely high value like the brightness of the sun (higher than the maximum screen brightness). Gamma correction was performed using a TES-1332A photo meter. Experiments were coded in MATLAB and were realized using Cogent Graphics toolbox developed by John Romaya at the LON at the Welcome Department of Imaging Neuroscience. The stimuli on each trial were composed of two homogenous, round $(1.2 \mathrm{~cm}$ diameter), temporally fluctuating gray patches with independently sampled gray-level on each monitor frame refresh. The two gray patches were presented on a black background and were positioned to the right and to the left of a fixation cross (total width from right edge of right patch to left edge of left patch: $5 \mathrm{~cm}$ ). The head position of the subjects was fixed at approximately $60 \mathrm{~cm}$ using a standard chinrest. For baseline trials, gray levels of the target and non-target were normally distributed around means of 0.4 and 0.3 (on a 0 to 1 (maximum screen 
brightness) scale in MATLAB) respectively. In Exp. 1, gray levels of the target and non-target stimuli for the multiplicative-boost condition were normally distributed around means of 0.6 and 0.45 respectively maintaining the $4 / 3$ ratio but increasing the difference from 0.1 to 0.15 compared with baseline. In the additive-boost condition, gray levels of the target and non-target were distributed around 0.6 and 0.5 , respectively, maintaining the difference of 0.1 but decreasing the ratio from 4/3 to 6/5 compared with baseline. In Exp. 2, the multiplicative-boost condition began the same as a baseline trial, but $100 \mathrm{~ms}$ into the trial both target and non target mean gray levels increased from 0.4 and 0.3 to 0.6 and 0.45 , respectively, maintaining the $4 / 3$ ratio but increasing the difference from 0.1 to 0.15 . The additive-boost condition was similar only that after $100-\mathrm{ms}$ gray levels of the target and nontarget increased from 0.4 and 0.3 to 0.6 and 0.5 , respectively, maintaining the difference of 0.1 but decreasing the ratio from $4 / 3$ to $6 / 5$. For all conditions in both experiments, on each frame the gray level for each individual patch was separately and independently recalculated as the sum of its designated mean plus a Gaussian random variable $\mathrm{N}\left(0,0.1^{2}\right)$. Occasional below threshold brightness samples might create the appearance of flickers where the stimulus appears to disappear and immediately reappear. Importantly, these flickers are more likely to occur for stimuli with lower mean brightness, potentially providing alternative strategies for performing the task (e.g., choose the one that flickers less). Thus, final brightness values below a value of 0.1 were truncated to 0.1 (a clearly visible, above threshold brightness value) to prevent obvious flickering of the stimuli that might attract attention to it in a bottom-up fashion. Because a brightness of 1 represents the maximum brightness afforded by the screen, final brightness values above 1 also were truncated to 1 . Refresh rate was set at $60 \mathrm{~Hz}$ (16.6 ms per frame) with new brightness levels independently resampled for each frame and for each stimulus location. Tests were run to evaluate the probability of dropped frames, and no frames were dropped after a full hour of continuous presentation. The location of the target (right/left) was randomly drawn on each trial.

\section{Procedure}

Responses were given on the 1 and 3 keys of the keyboard number keypad for the left and right responses, respectively. Subjects were instructed to use the right index finger for the 3 key and the same finger on the left hand for the 1 key. The stimuli stayed on until the response was entered, after which a 1-s Inter-Stimulus-Interval (ISI), preceded the next trial. All trials were randomly assigned to one of three possible conditions: baseline, multiplicative boost, and additive boost. Participants were presented with 10 blocks of 60 trials per session for a total of 1,200 trials per participant. Each block consisted of $40 \%, 30 \%$, and $30 \%$ of randomply intermixed baseline, multiplicative boost, and additive boost trials, respectively. Feedback on error responses was provided in the form of a 1-second loud beep immediately following the response. No feedback was provided after correct responses. After each block, there was a self-timed intermission to allow the subject to rest. During each of these breaks, the average accuracy and RT for the last block were presented on the screen. The participants were instructed to try to maximize their performance (try to respond as fast and as accurately as possible), such that if they reached $100 \%$ accuracy they should try to respond faster and were given a 30 -trial practice block. Subjects also were told to keep their eyes focused on the fixation cross throughout the trial. Maintaining a constant fixation facilitates performance of the task by allowing simultaneous monitoring of both patches, while maintaining constant retinotopic mapping between stimulus and response. Nevertheless, in the absence of an eye tracker, there was no way to verify that they actually complied with this request. The experiment was held in a partially darkened room, and subjects were acclimated to the lighting conditions during the practice.

\section{Results}

\section{Experiment 1 results}

The intensity manipulations in Exp. 1 yielded significant effects on both correct RT $(\mathrm{F}(2,12)=10.676, p<0.01$; repeated measures ANOVA) and accuracy $(\mathrm{F}(2,12)=23.81, p<0.0001$; repeated measures ANOVA. RT and accuracy results are illustrated in Fig. 1c. A post-hoc analysis (Tukey HSD test) of the accuracy data revealed that subjects were less accurate in the additive boost condition $(M=0.77, S D=0.04)$ compared with both the baseline $(M=0.86, S D=0.04 ; p<0.001)$ and multiplicative boost condition $(M=0.86, S D=0.04 ; p<0.001)$. Accuracy levels in the baseline and multiplicative boost conditions were practically identical $(p=1)$. A post-hoc analysis (Tukey HSD test) of correct RTs revealed that compared to baseline condition $(M=0.95 \mathrm{~s}, S D=0.19)$ subjects responded faster in both the additive boost condition $(M=0.87 \mathrm{~s}, S D=$ $0.16 ; p<0.02)$ and the multiplicative boost condition $(M=$ $0.83 \mathrm{~s}, S D=0.15 ; p<0.01$ ). In a previous study (Teodorescu and Usher 2013), we found slower RTs in a condition similar to the additive boost compared with a condition similar to the multiplicative boost. On this basis, we performed a planned comparison for this contrast alone and indeed found slower RTs in the additive compared with the multiplicative condition $^{2}(\mathrm{~F}(1,6)=7.75, p<0.05$, planned comparison).

\footnotetext{
${ }^{2}$ Note that this contrast was not excluded from the Tukey HSD test. Thus, our Tukey HSD statistics represent a conservative significance test.
} 


\section{Experiment 2 results}

The manipulation yielded significant effects of both correct RT $(\mathrm{F}(2,12)=35.96, p<0.00001$; repeated measures ANOVA $)$ and accuracy $(\mathrm{F}(2,12)=21.67, p<0.001$; repeated measures ANOVA; Fig. 1c). A post-hoc analysis of the accuracy data revealed that subjects were less accurate in the additive boost condition $(M=0.81, S D=0.088)$ compared with both the baseline ( $M=0.88, S D=0.056 ; p<0.001$; Tukey HSD test) and multiplicative boost condition $(M=0.88, S D=0.082$; $p<0.001$; Tukey HSD test). Accuracy in the baseline and multiplicative boost conditions were nearly identical ( $p=0.97$; Tukey HSD test). A post-hoc analysis of the RT data revealed that compared to baseline ( $M=1.004 \mathrm{~s}, S D=0.188)$ subjects responded faster in both the additive boost condition ( $M=$ $0.914 \mathrm{~s}, S D=0.175 ; p<0.001$; Tukey HSD test) and the multiplicative boost condition ( $M=0.860 \mathrm{~s}, S D=0.157$; $p<0.001$; Tukey HSD test). Last, as in Exp. 1, consistent with our previous study (Teodorescu and Usher 2013), we also found slower RTs in the additive compared with the multiplicative condition $(\mathrm{F}(1,6)=9.59 ; p<0.05$; planned comparison).

\section{Discussion of experimental results}

Strikingly, these findings demonstrate that both common forms of invariance predicted by purely relative models were violated. For RT, invariance held for neither equi-difference nor equi-ratio intensities (faster RTs in both boost conditions compared to baseline). For accuracy however, intensity invariance was violated with respect to differences (lower accuracy in the additive boost compared to the baseline condition) but not with respect to ratios (equal accuracy in the multiplicative boost compared to baseline condition). As we will show below (Computational Modeling section), neither the pure DDM nor the pure normalization model can capture these qualitative patterns.

Additionally, comparing the additive and the multiplicative conditions, we note that the brighter channel is identically distributed in both conditions (mean $=0.6$ ), whereas the dimmer channel is, on average, brighter in the additive boost condition (0.5) than in the multiplicative boost condition (0.45). The finding of slower RT's in the additive versus multiplicative boost condition indicates competition and thus violates a fundamental prediction of independent race models (Teodorescu and Usher 2013). Independent race models, are "purely absolute" in that they are driven solely by absolute input values in contrast to competitive models, in which the interaction between the input values also contribute to the integrated evaluations (Teodorescu and Usher 2013). Thus, the current results speak against "model purity" either in the relative or the absolute sense. As we show in the following modeling section, only "hybrid" models, which maintain sensitivity to both the relative and absolute aspects of the stimuli, can satisfactorily account for our findings. To anticipate our computational modeling results, we find two ways of accounting for the joint observation of absolute and relative processing. The first is an LCA account, which combines absolute and relative evidence dynamically, and the second is a diffusion account, in which the stopping rule is based on differences (thus relative) and a separate component, the processing noise, increases with the magnitude of the input (thus absolute).

\section{Computational modeling}

Our results indicate a need for computational models of decision making to possess mechanisms for absolute value sensitivity in addition to relative processing. In this section, we investigate how different sources of sensitivity to absolute values interact with different relative decision processes. To this end, we first consider potential sources for absolute value sensitivity that are independent of the modeling framework. We then proceed to explore three model families. The first two originate from a purely relative conceptualization of the decision process based on either ratios (as in the normalization model) or differences (as in the DDM model). Within each of these families, we consider both a purely relative version and a hybrid version where relative processing is not pure but varies parametrically on a continuum between purely absolute and purely relative extremes. We explore a third model family, dynamic relativity (as in the LCA model; Teodorescu and Usher 2013; Usher and McClelland 2001), which is of an intrinsically hybrid nature. The LCA model is related to differential relativity, but here relativity emerges dynamically over time as a result of lateral inhibition. We then test the ability of the different models to account for our results by fitting the models to empirical joint distributions of response probability, correct RT, and error RT from Exp. 1. We conclude by discussing insights from the model fitting exercise and explore model predictions for hypothetical input spaces.

\section{Value sensitivity}

Three sources for sensitivity to absolute values are examined. The first two are general in the sense that they are independent of specific model assumptions and thus can be implemented in any model. These sources relate to the well-established observation that, for above threshold values, sensitivity to equal differences decreases with intensity according to psychophysical laws. Lighting the 111 th candle makes less of a difference than the 11th, and people will drive farther for a $\$ 30$ discount on $\$ 100$ than for the same discount on a $\$ 1,000$ product. At the most basic level, this effect has been attributed to 
constant processing noise on top of a compressive, nonlinear relation between objective values (e.g., physical, numerical) and their corresponding representation as neural activations (Geisler 1989). To capture the nonlinear relationship between the momentary intensity at each stimulus location (i.e., separately for brightness fluctuations of the right and left gray patches in our paradigm) and their corresponding internal representations, the momentary input values $I_{i}(t)$ feeding the decision process were modeled as:

$I_{i}(t)=S_{i}(t)^{\gamma}$

$S_{i}(t)$ has the same distribution as the momentary physical stimulus value (brightness) at location $i$ (left/right) and $\gamma>0$ is a power coefficient representing the nonlinear nature of the perceptual (or neuronal) transformation. ${ }^{3}$

Diminishing value sensitivity also can be accounted for by assuming that the processing of higher neural activations is accompanied by higher variability (Geisler 1989). The segregation of processing noise into two components: one for constant general noise and another for activation dependent, multiplicative noise has been instrumental in several recent studies (Brunton et al. 2013; Louie et al. 2013; Lu and Dosher 2008). To test this relation, processing variability $\xi_{i}(t)$ in channel $i$ was modeled as a function of input intensity $I_{i}(t)$ according to:

$$
\xi_{i}(t) \sim N\left(0,\left(\pi I_{i}(t)\right)^{2}\right)+N\left(0, \sigma^{2}\right)=N\left(0,\left(\pi I_{i}(t)\right)^{2}+\sigma^{2}\right)
$$

$\sigma$ stands for the magnitude of general, stimulus independent processing noise, and $\pi$ represents the sensitivity of stimulus dependent input noise to input intensity. ${ }^{4}$ Multiplicative noise and psychophysical transformations introduce value sensitivity at the level of the input and thus are independent from assumptions about the type of information processing relativity in the decision mechanism.

Computational choice-RT models traditionally have been pure in the sense that they were either purely absolute (independent race model; Usher, Olami \& McClelland, 2002; Van Zandt et al. 2000; Vickers 1970) or implemented difference and ratio relations in their pure form. However, purely relative and purely absolute processes do not represent discrete states but rather two extremes on a continuum. The balance between absolute and relative processing can be parameterized to interpolate between the two states. The form of this interpolation depends on the type of information

\footnotetext{
${ }^{3}$ Note that only clearly visible, above threshold brightness values were used in the experiments. Thus, in Eq. 1 and all that follow, the perceptual threshold value for brightness and the unique dynamics of sub-threshold brightness perception are neglected.

${ }^{4}$ A model corresponding to the Poisson assumption with $\xi_{i}(t) \sim N(0$, $\left.\pi I_{i}(t)+\sigma^{2}\right)$ also was tested but did not perform as well.
}

processing relativity assumed in the decision stage. Thus, this third mechanism for sensitivity to absolute values will be treated separately for each model family in the following three sections.

\section{Fractional relativity: a normalization model analysis}

To test the fractional relativity assumption, we implement a full normalization model (Louie et al. 2013) where a decision is made when one of the accumulated normalized inputs (i.e., input ratios) $X_{i}$ crosses a predetermined threshold. The dynamics of $X_{i}$ are described by:

$$
\left\{\begin{aligned}
X_{i}(t+1) & =X_{i}(t)+I_{i}^{N}(t)+\xi_{i}(t) \\
I_{i}^{N} & =\frac{I_{i}(t)}{\lambda+\sum I_{i}(t)}
\end{aligned}\right.
$$

$I_{i}(t)$ is the momentary input (Eq. 1), $I_{i}^{N}(t)$ is the normalized momentary input, $\xi_{i}(t)$ is the momentary processing noise, independent over time and across channels (Eq. 2), and the semi-saturation parameter $\lambda \geq 0$ allows the model to transition continuously between a purely relative ratio (or normalization) model $(\lambda=0)$ and an asymptotically purely absolute independent race model $\left(\lambda \gg \sum l\right)$.

\section{Differential relativity: a diffusion model analysis}

We conceptualize the DDM as two independent accumulators with the stopping rule applied to the difference (for a recent application of this algorithm to simultaneous modeling of both perceptual and economic decisions, see Towal et al. 2013). This architecture allowed us to model the flow of information from the momentary perceptual values of each stimulus alternative (right and left brightness patches) through two separate, parallel processing channels. Thus, a decision is made when the momentary difference in accumulated evidence $D(t)$ crosses a predetermined threshold. For two alternative choices this process is described by:

$\left\{\begin{array}{c}D(t)=\max \left\{X_{i}(t)\right\}-\alpha \min \left\{X_{i}(t)\right\} \\ X_{i}(t+1)=X_{i}(t)+I_{i}(t)+\xi_{i}(t)\end{array}\right.$

$D(t)$ is the relative decision module tracking the momentary differences between the accumulated evidence in favor of the two response alternatives, $0 \leq \alpha \leq 1$ is the partial relativity coefficient that allows the model to transition between a purely relative DDM ( $\alpha=1$ implies: stop when the difference crosses a threshold) and purely absolute independent race model ( $\alpha=0$ implies: stop when the largest independent accumulator crosses a threshold; cf. Moreno-Bote 2010; Zylberberg et al. 2012), 
$X_{i}(t)$ are the accumulated evidence values at time $t$ corresponding to the target and non-target alternatives and $\xi_{i}(t)$ is the momentary processing noise, independent over time and across channels (Eq. 2).

\section{Dynamic relativity: a leaky-competing accumulator model analysis}

The Leaky Competing Accumulator model (Bogacz et al. 2007; Usher and McClelland 2001) provides a different approach to partially relative information processing, and variants of this model have been used to study decision making in both humans (Teodorescu and Usher 2013) and social insect colonies (Marshall et al. 2009). The LCA model is based on a basic race architecture but assumes in addition that at each time step, accumulators lose information (neural leak) and inhibit each other, both in proportion to the accumulated activation value in each processing channel. Thus, the accumulators are described by the equation:

$X_{i}(t+1)=\max \left\{0, k X_{i}(t)+I_{i}(t)-\beta \sum_{j \neq i} X_{j}+\xi_{i}(t)\right\}$

$0<\mathrm{k}<1$ is the proportion of activation that remains after neural leak, $\beta$ is the proportion of lateral inhibition between accumulators, and the max function is a neurally inspired nonlinearity preventing neural activation from attaining negative values. Because inhibition is proportional to total activation, it plays only a minor role early in the trial (when activation levels are still low compared with the absolute input values), approximating an independent race. However, as activation is accumulated, the amount of inhibition in the system increases until it overshadows the absolute input values and leads to diffusion-like ${ }^{5}$ process that, granted enough time, terminates with "winner-take-all" dynamics (Bogacz et al. 2006; Marshall et al. 2009). Whereas one accumulator continues to grow (asymptotically limited by leak), the other is suppressed towards zero (see Fig. 2 for example LCA trajectories for the additiveboost manipulation).

\section{Modeling methods}

The present section deals with practical, theoretical, and experimental assumptions required for computational modeling

\footnotetext{
${ }^{5}$ More technically, under certain assumptions, the LCA can be mathematically decomposed into two components $Y_{1}=X_{1}+X_{2} \& \quad Y_{2}=X_{1}-X_{2}$ (Bogacz et al. 2006; Heathcote 1998; Marshall et al. 2009). The former $\left(Y_{1}\right)$ is an absolute component predominant in the initial stages of accumulation, the latter $\left(Y_{2}\right)$ is a differential relativity component dominating the final stages. The absolute component $Y_{1}$ represents the speed with which the process approaches the threshold in the initial stagees of accumulation which will be achieved faster when absolute input values are higher. Thus, the LCA also can be conceptualized as a DDM-like process with lower thresholds for conditions with higher absolute input values.
}

and fitting of choice RT data in general and for this study in particular. The first subsection, "Modeling Assumptions," is less technical and is instructive with regards to the nature of the relation between experimental design and computational modeling. The rest of the subsections, however, are more technical and can be safely skipped, either entirely or partially. Those not interested in the technical details presented in this section can continue reading from the Modeling Results section.

\section{Modeling assumptions}

In the following model fits, input values were sampled from the same distributions used to generate the stimuli in the experiment $\left(S_{i}(t)\right.$ Eq. 1), so that the models "experienced" the exact same external environment as the subjects. This constrained approach to modeling the experimental setting is crucial in discriminating between models that could otherwise closely mimic each other (Teodorescu and Usher 2013), especially in view of recent concerns regarding the falsifiability of response-time models (Jones and Dzhafarov 2013). However, constraining model inputs to the same distribution as the physical stimulus intensities is a nontrivial assumption that may be unsuited for many experimental paradigms. In fact, the common practice when fitting choice RT models to data is to allow input values to vary freely between conditions and assign free parameters for each input and condition combination. What allows us to assume a more constrained relationship between physical stimulus values and input strengths relates directly to the choice of stimuli and the design of the experimental paradigm, which in turn are motivated by neural constraints. In our design, the stimuli are such that predecisional neural interactions between the channels are minimized. This is achieved by choosing a low-level perceptual modality (brightness or contrast is arguably the lowest level of visual processing) while maintaining spatial separation between the perceptual evidence streams (for a comprehensive discussion see Teodorescu and Usher 2013).

In addition, the use of highly overlapping, temporally variable stimuli distributions, intermixed within blocks justifies the assumption of constant decision thresholds between experimental conditions. A common selective influence assumption is that under such conditions, only drift-rates should be allowed to vary between conditions (Ratcliff and Smith 2004). However, having the model inputs linked directly to the physical properties of the stimuli allowed us to derive (rather than fit) condition dependent drift-rates (Teodorescu and Usher 2013). All of this resulted in zero free parameters being allowed to vary between experimental conditions. Model freedom was confined to general model parameters, thus forcing the models to predict the pattern of results for all experimental conditions with a single unique set of parameters. 


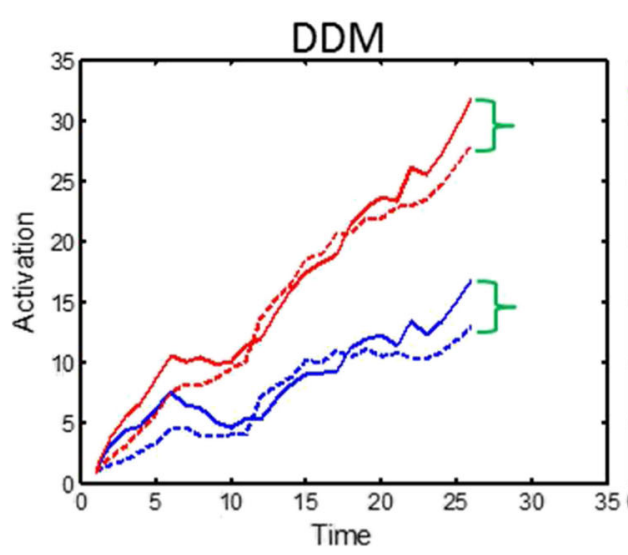

Fig. 2 Baseline vs. additive boost activation trajectories for the target and non-target accumulators $\left(\mathrm{X}_{1} \& \mathrm{X}_{2}\right.$, respectively) with identical noise sequences for representative models. Left: DDM. Center: independent race model; Right: LCA model. The green lines represent the absolute thresholds on accumulation, and the green parentheses represent the

Note that these constraints do not imply that drift rates were fully determined by the physical stimulus values. As mentioned earlier, parametric assumptions were introduced into all the models regarding the psychophysical transformations of physical (energy) values into psychological (perceived) values (Eq. 1) as well as the dependence of processing noise on stimulus intensity (Eq. 2). In addition, assumptions about the relationships between inputs, such as constraining the sum of all drift-rates to equal a constant, also were captured parametrically by incorporating continuous partial relativity parameters. Having drift-rates constrained by the physical properties of the stimuli also allowed us to use computational simulations, based on the results of the model fits, to generate input space mappings, which produce model predictions for a continuum of possible empirical manipulations.

\section{Model parameters}

\section{Standard parameters}

Following the above experimental and theoretical considerations, no parameters in our models were allowed to vary between experimental conditions. Specifically, 4 standard free parameters were included with all models: (1) threshold (Th) that represents response caution such that higher thresholds lead to slower but more accurate decisions; (2) general processing noise $\left(\sigma^{2}\right)$ representing the intrinsically noisy nature of neural processing; (3) nondecision time $\left(\mathrm{T}_{\mathrm{nd}}\right)$ corresponding to the time it takes to process all nondecision components, such as perceptual encoding and response generation processes; and (4) a time-step parameter $\left(T_{s}\right)$, which is a scaling parameter that determines the equivalent duration in milliseconds for one computational iteration. The last two parameters are needed to transform all simulated RTs (RT'; expressed as discrete
LCA

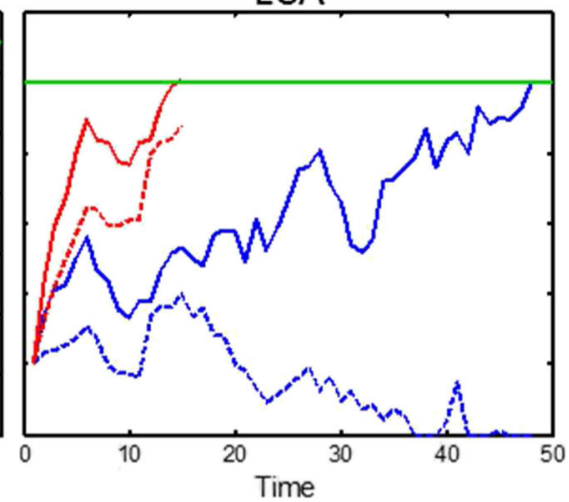

relative threshold on the difference between accumulated evidence. While the independent race and LCA reach the threshold faster for higher but equi-difference absolute inputs, the DDM is invariant to this manipulation

time steps) to real-world RTs (expressed in milliseconds) such that $\mathrm{RT}=\mathrm{T}_{\mathrm{s}} * \mathrm{RT}^{\prime}+\mathrm{T}_{\mathrm{nd}}$.

In addition, all models included two parameters that describe the dependence of model inputs on the physical properties of the stimuli. Momentary drift-rates $I_{i}(t)$ for each channel $(\mathrm{I}=$ left/right) were directly derived from the momentary physical brightness values $S_{i}(t)$ by transforming them through a psychophysical power law (Eq. 1) and perturbing the ensuing value by intensity dependent random variability (Eq. 2). The psychophysical transformation required one parameter, a power-law coefficient $(\gamma)$ representing the concavity of the psychophysical transformation (Eq. 1). The intensity dependent random input variability required an additional parameter, an input-noise coefficient $(\pi)$, representing the sensitivity of the standard deviation of stimulus dependent input-noise to the momentary perceived stimulus intensity (i.e., post psychophysical transformation; Eq. 2).

\section{Between-trial variability parameters}

In order for sequential sampling models to be able to describe correctly the form of both correct and error RT distributions, it is common practice to add some sources of between-trial variability. One source of between-trial variability, starting point variability (SPV), was applied to all models in the same manner. In all our simulations, the accumulation process for each alternative $i$ began at an arbitrary baseline activation level of 0.4 to which we added a uniformly distributed random variable U(0, SPV) independent across channels. All models, except for the LCA, also traditionally require an additional between-trial drift-rate variability parameter $(\eta)$ to account well for the form of response time distributions (Ratcliff and Rouder 1998). This was implemented in the models as a Gaussian random variable $\mathrm{N}\left(0, \eta^{2}\right)$ that is drawn once for each trial and independently for each channel $\mathrm{i}$ and added to all 
inputs $I_{i}$ for the duration of that simulation trial. The normalized race model predicted distributions that were too symmetrical, so following Ratcliff and Smith (2004) and Teodorescu and Usher (2013) we augmented it with a parameter for between trial exponential variability in response criteria $(\tau)$ such that for each trial, response criteria were drawn from an exponential distribution $T h+\exp (\tau)$.

\section{Relativity parameters}

To control the degree of processing relativity in the normalization and DDM models, we also included a partial-relativity parameter, which was implemented differently for the normalization and DDM models (see $\lambda \& \alpha$ in Eqs. 3 and 4, respectively). The LCA model also required two additional parameters for leak $(\mathrm{k})$ and inhibition $(\beta)$. The inhibition parameter $(\beta)$ is considered the partial relativity parameter for the LCA model, because in its absence $(\beta=0)$ the model reduces to a purely absolute, independent, leaky race model. Note, however, that the LCA is fundamentally value sensitive and it does not have a purely relative form. For the LCA, some sensitivity to absolute input values is retained early in the process for any value of $\beta$.

\section{General optimization algorithm}

To evaluate the models' ability to account for response time distributions and response accuracy, we fit the models to the empirical data of Experiment 1. Models were fit to individual participant data as well as to aggregate dada representing the average observer. To optimize model parameters and to determine how well the models fit the data, we used Quantile Maximal Likelihood (QMPE; Heathcote et al. 2004; Heathcote and Brown 2004). In QMPE, for each model, the parameter space is searched for a set of parameters that maximizes the likelihood of the empirical data once precise RT values are censored to bins defined by RT quantiles (see below). Technically, this search was achieved by using the SIMP LEX algorithm (Nelder and Mead 1965) as implemented by the "fminsearch" function in MATLAB. The full algorithm consisted of repeated simplex optimizations with 10,000 iterations per model evaluation, the first beginning with a random starting point, and each ensuing simplex beginning with the parameter set obtained by the previous simplex. This process continued for as long as the goodness of fit (likelihood) improved. The process terminated at the first occurrence of a simplex optimization, which failed to improve on the likelihood achieved by its predecessor. The parameters from the last optimization are likely to be the result of a random drift away from the local minima due to the stochastic nature of the models. These parameters were therefore discarded, and the one-but-last set of parameters was used as the starting point of a final "fine-tuning" simplex optimization with 100,000 iterations per model evaluation. The "finetuned" parameter set was retained for model comparison purposes. For each model, the above procedure was repeated with 10 starting points for the group (aggregate) data set and 5 starting points for each of the individual data sets, yielding 10 candidate-parameter sets for the group data and 5 for each of the participants. The reported best fitting parameters (Tables S1 \& S2, Supplementary Materials) are the ones achieving the best average likelihood out of the identified candidates. Average likelihood was calculated for each candidate parameter set based on 20 repeated simulation runs. Starting points for the group data fits were generated by initially finding a reasonable parameter set for each model and then taking random values between $60 \%$ and $140 \%$ of the original parameters. Starting points for the individual fits were generated in a similar manner only with the best fitting parameters identified at the group level acting as the initial parameter set. While some logical and theoretical constraints apply to the final parameter values, no constraints were imposed on parameter values during the optimization procedure, allowing parameters to assume values outside the original range of the starting points. This method provided a reasonable compromise between computational complexity and fit quality. Using this method, we achieved good fits for all models except for the fits to individual data for the most complex model (the full normalization model; model 2 in Tables 1 and 2). Due to the higher complexity, this model had difficulties converging for some of the individual subjects. For this model, additional explorations were conducted with different starting points, including the best-fitting (group as well as individual) parameters for a submodel (the pure normalization model; model 1, Tables 1 and 2) The final performance of this model was evaluated according to the best parameter sets identified over all optimization results.

\section{Error function}

To generate the QMPE, for each of the seven participants, we collapsed across right and left responses ${ }^{6}$ and calculated the $0.1,0.3,0.5,0.7$, and 0.9 RT quantiles and response proportions for each of the three experimental condition (baseline/ additive boost/multiplicative boost) and for each of the two response types (correct/error) resulting in 6 sets of 5 quantiles for each participant (a total of 2 (correct/error) * 3 (condition) $* 5=30$ quantiles). Thus, for each participant and for each condition and response type combination, six bins can be defined using the 5 quantiles as borders. To estimate model

\footnotetext{
${ }^{6}$ The experiments were fully randomized, containing exactly the same number of right and left trials. In addition, feedback was the same for right and left trials and no rewards were provided. Thus, no bias for right or left response was expected and consequently all data were collapsed over right and left responses.
} 
Table 1 Model goodness of fit scores for group data

\begin{tabular}{|c|c|c|c|c|r|r|r|}
\hline Model \# & Relativity & Noise & ParNum & LogL & SD(LogL) & BIC & AIC \\
\hline $\mathbf{1}$ & Pure Normalization & constant & 8 & 37188 & 11.15 & 37260 & 37204 \\
\hline $\mathbf{2}$ & Partial Normalization & proportional & 10 & 37075 & 11.85 & 37165 & 37095 \\
\hline $\mathbf{3}$ & Pure DDM & constant & 7 & 37110 & 9.73 & 37173 & 37124 \\
\hline $\mathbf{4}$ & Partial DDM & proportional & 9 & 36923 & 5.41 & 37004 & 36941 \\
\hline $\mathbf{5}$ & LCA & proportional & 9 & 36917 & 5.04 & 36998 & 36935 \\
\hline
\end{tabular}

Mean likelihoods and SDs are based on 20 repeated simulations for each model with the best fitting parameters (100,000 iterations per simulation). AIC and BIC are calculated based on the mean likelihoods with $N=8,400$ (the total number of observations over all subjects). The colors indicate normalized magnitude within each column (green indicates smaller values - better fit or lower variability for the SD column; red indicates larger values - poorer fit or higher variability for the SD column). Note that the LogLikelihood values obtained for models 1, 2, and 3 are higher than either the AIC or the BIC values obtained for models 4 and 5. In other words, because penalty for complexity in AIC and BIC is added to the LogLikelihood value, these results demonstrate that models 1,2 , and 3 would have performed worse than models 4 and 5 even if they had no free parameters. This result renders exploration of simpler, submodels of models 1,2 , and 3 unnecessary

predictions for a given set of parameters, we simulated 10,000 or 100,000 (for fine tuning) trials and calculated, for each of the experimental conditions, the proportion of trials that fall within the bounds of each of the 12 bins corresponding to choice accuracy and RT quantiles (6 bins for correct response and 6 for errors; note that for each condition these 12 proportions sum to 1 ). Denoting the number of empirical observations in a particular bin $i$ by $n_{i}$ and the probability predicted by the model for a particular bin $\mathrm{i}$ by $\mathrm{P}_{\mathrm{i}}$, the likelihood $\mathrm{L}$ of the data given the model is defined as:

$$
L=\prod_{i} P_{i}^{n_{i}}
$$

where $i$ rangers over the entire set of 36 bins. In addition to fitting the models to individual participants, we fit the models to "average observer data." For the average observer data, quantiles were calculated by averaging the corresponding individual participant quantiles and bin frequencies were obtained

Table 2 Model goodness of fit scores for individual participants

\begin{tabular}{|c|c|c|c|c|c|c|c|c|}
\hline \multirow[b]{2}{*}{$\#$} & \multirow[b]{2}{*}{ Model } & \multicolumn{7}{|c|}{ Individual Subjects (LogL) } \\
\hline & & \#1 & $\# 2$ & \#3 & $\# 4$ & \#5 & \#6 & $\# 7$ \\
\hline 1 & Pure Normalization & 5207 & 5135 & 5519 & 5366 & 5698 & 5406 & 5462 \\
\hline 2 & Partial Normalization & 5204 & 5134 & 5496 & 5282 & 5637 & 5389 & 5417 \\
\hline 3 & Pure DDM & 5211 & 5137 & 5477 & 5289 & 5713 & 5402 & 5425 \\
\hline 4 & Partial DDM & 5148 & 5114 & 5440 & 5284 & 5554 & 5376 & 5402 \\
\hline \multirow[t]{2}{*}{5} & LCA & 5174 & 5100 & 5450 & 5293 & 5516 & 5345 & 5401 \\
\hline & & \multicolumn{7}{|c|}{ Individual Subjects (BIC) } \\
\hline \# & Model & \#1 & $\# 2$ & \#3 & $\# 4$ & \#5 & $\# 6$ & \#7 \\
\hline 1 & Pure Normalization & 5264 & 5192 & 5575 & 5423 & 5755 & 5462 & 5519 \\
\hline 2 & Partial Normalization & 5275 & 5205 & 5567 & 5353 & 5708 & 5460 & 5488 \\
\hline 3 & Pure DDM & 5261 & 5186 & 5526 & 5339 & 5763 & 5451 & 5474 \\
\hline 4 & Partial DDM & 5212 & 5178 & 5504 & 5347 & 5618 & 5440 & 5466 \\
\hline \multirow[t]{2}{*}{5} & LCA & 5238 & 5164 & 5514 & 5356 & 5580 & 5409 & 5465 \\
\hline & & \multicolumn{7}{|c|}{ Individual Subjects (AIC) } \\
\hline \# & Model & $\# 1$ & $\# 2$ & \#3 & $\# 4$ & \#5 & $\# 6$ & \#7 \\
\hline 1 & Pure Normalization & 5223 & 5151 & 5535 & 5382 & 5714 & 5422 & 5478 \\
\hline 2 & Partial Normalization & 5224 & 5154 & 5516 & 5302 & 5657 & 5409 & 5437 \\
\hline 3 & Pure DDM & 5225 & 5151 & 5491 & 5303 & 5727 & 5416 & 5439 \\
\hline 4 & Partial DDM & 5166 & 5132 & 5458 & 5302 & 5572 & 5394 & 5420 \\
\hline 5 & LCA & 5192 & 5118 & 5468 & 5311 & 5534 & 5363 & 5419 \\
\hline
\end{tabular}

Mean likelihoods and SDs are based on 20 repeated simulations for each model with the best fitting parameters (100,000 iterations per simulation). AIC and BIC are calculated based on the mean likelihoods with $\mathrm{N}=1,200$ (the total number of observations per subjects). The colors indicate normalized magnitude within each column (green indicated smaller values - better fit; red indicates larger values - poorer fit) 
by summing the corresponding bin frequencies across participants (Moran et al. 2013).

The objective of the optimization procedure was to find, for each model, a parameter set that maximizes the likelihood L of the data. In practice, this was achieved by minimizing twice the negative log-likelihood:

$-2 \ln (L)=-2 \sum_{i} n_{i} \ln \left(P_{i}\right)$

Optimizing the models to predict binned response proportions allows us to test the models on their ability to simultaneously account for both RT distributions of correct and error responses as well as accuracy (i.e response probabilities).

\section{Model comparison}

We fit the models to both group (average observer) and individual participant data. The results are reported in Tables 1 and 2, respectively. Because model comparisons based on individual fits are in close agreement with group, average observer fits, in the remainder of the paper we focus our discussion on the model comparisons based on fits to group data. This choice is motivated by our desire to focus the discussion on general qualitative differences between the models rather than on individual differences (Ratcliff and Smith 2004).

\section{AIC and BIC scores}

To compare model performance, we provide two goodness of fit measures that penalize models for extra complexity: (1) The Bayesian Information Criterion (BIC; Schwarz 1978); and (2) Akaike Information Criterion (AIC; Akaike 1974). Complexity, in these methods, is operationalized as proportional to the total number of free parameters and the two methods differ with respect to the magnitude of this penalty. Generally, AIC being more liberal and BIC more conservative.

BIC was calculated as:

$B I C=-2 \ln (L)+k \ln (N)$

and AIC was calculated as:

$A I C=-2 \ln (L)+2 k$

where $k$ is the number of free parameters for each model. $N$ is calculated differently for the average observer and individual subject fits. For the average observer, $N$ is the total number of trials across all participants, whereas for each individual participant, $N$ is the total number of trials for that specific participant. The BIC is a relative measure where a difference in scores that is greater than 10 can be considered substantial (Raftery 1995).

\section{Modeling results}

\section{Fractional relativity}

In its purely relative form $(\lambda=0)$ and in the absence of intensity dependent noise and psychophysical transformations $(\pi=0 ; \gamma=1)$, fractional relativity would predict complete invariance to the multiplicative boost manipulation. We first fit the purely relative model with flexibility in the form of the psychophysical transformation $(\gamma \geq 0)$ to the data from Exp. 1. In this form, the model captured the accuracies of the different conditions well but missed all RT effects, both quantitatively and qualitatively (Tables 1 and 2, Model 1; Fig. 3 left column; for all models see SI Figure S1 for Quantile Probability Plots (QPF; Ratcliff and Smith 2004) describing the full choice and RT distributions for correct and error responses for the best average observer fitting parameters of all models; see also Table S2 for average observer best fitting parameters and Table S1 for individual participant best fitting parameters). To test the roles of the three sources of absolute value sensitivity within the fractional relativity framework, we fit the full normalization model (Eq. 3). This form (i.e., $\lambda \geq 0 ; \pi \geq 0 ; 0 \leq \gamma \leq 1$ ) achieved better results (Tables 1 and 2, Model 2), but for the average observer this was obtained with $\lambda \cong 79 \gg \sum I$ indicating no role for normalization (i.e., asymptotically independent race model; Fig. 2, central panel, for example trajectories of an independent race model demonstrating the RT speedup effect). While naturally sensitive to absolute input values, independent race models cannot capture the slower responses in the additive compared with the multiplicative-boost condition (Teodorescu and Usher 2013). Even with all forms of value sensitivity, the normalization model was not able to simultaneously capture all the qualitative patterns in our data. Thus, our results support neither form of fractional relativity (pure or partial) nor purely absolute processing.

\section{Differential relativity}

In its most widely used form the DDM is purely relative $(\alpha=1)$, and in the absence of intensity-dependent noise and psychophysical transformations $(\pi=0 ; \gamma=1)$ would predict complete invariance to the additive boost manipulation (see Fig. 2, left panel for example trajectories demonstrating this). Fitting the purely relative model with $\gamma$ free to vary, the best fit was achieved with $\gamma=0.49<1$ (typically $\gamma \sim 0.5$ for brightness stimuli; Geisler 1989) leading to a compressive psychophysical transformation. Thus, the DDM "perceived" the brightness difference between the two alternatives ${ }^{7}$ in the, high intensity, additive-boost condition as

\footnotetext{
7 "Perceived" difference is equivalent to drift-rate in the classic formulation of the DDM model.
} 


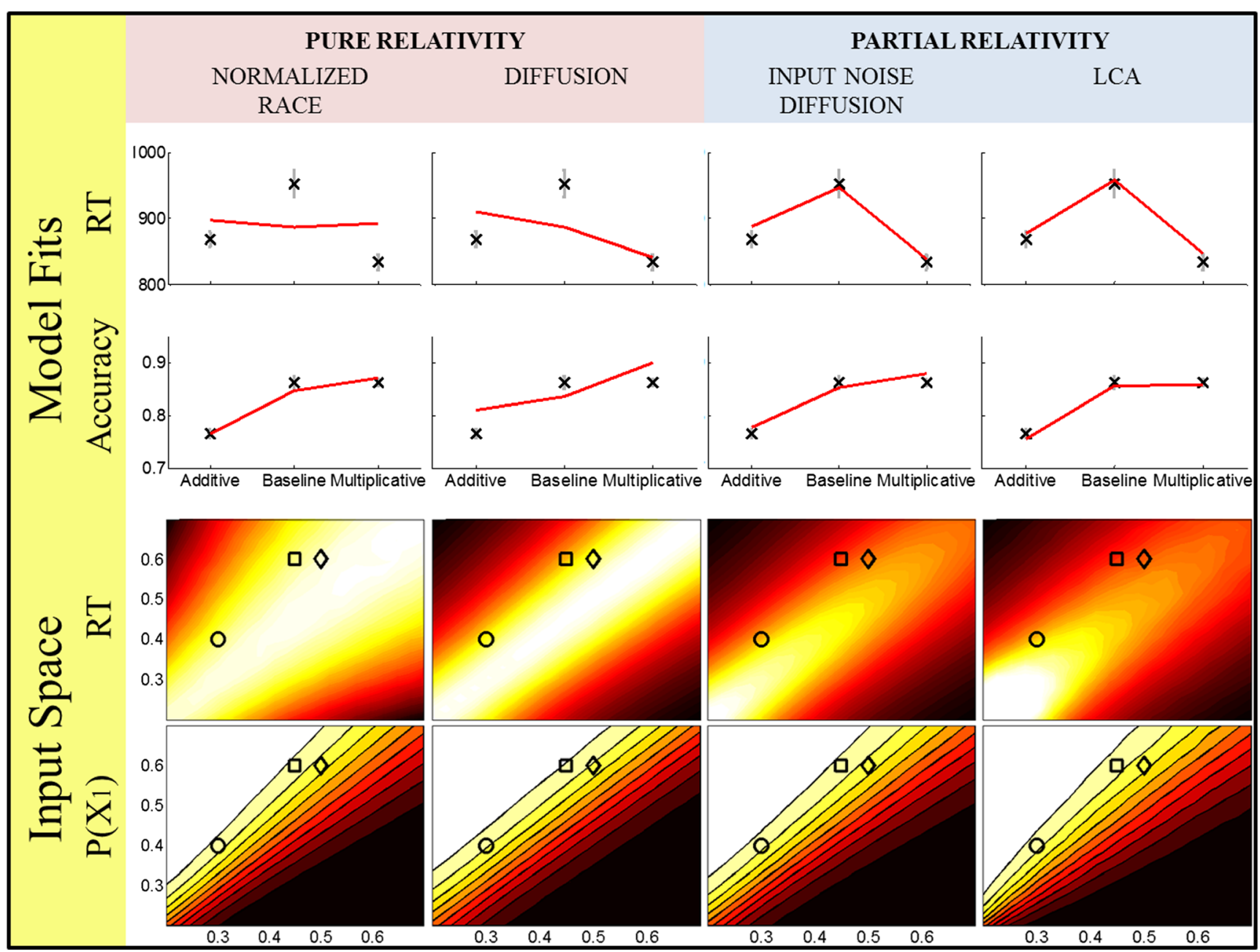

Fig. 3 Top: mean RT (upper panel) and accuracy (lower panel) model fits $($ models $1,3,4$, and 5 , Table 1$)$ to group data from Exp. $1($ red lines $=$ model; black ' $x$ 's $=$ data, with gray error bars representing within subject standard errors according to (Cousineau 2005). While model fits were performed on quantile data to simultaneously capture accuracy and RT distributions (for correct and error trials), only mean overall RT and accuracy are shown to highlight the qualitative effects. Bottom: Model predictions for $2 \mathrm{AFC}$ perceptual input spaces $\left(\mathrm{I}_{1}=\mathrm{Y}\right.$ axis; $\mathrm{I}_{2}=\mathrm{X}$ axis $)$ based on simulations with the best fitting parameters for each model (as in Table S1). The upper panel corresponds to mean overall RT and the bottom panel to the proportion of I1 responses (white $=$ high values; black = low values; to maximize the visibility of the contours, the color range was normalized separately for each panel and all RT values> $1,100 \mathrm{~ms}$ were trimmed). The circle, square, and diamond markers respectively represent the mean brightness values used in the baseline, multiplicative-boost, and additive-boost conditions in experiment 1 . Contour lines are only included in the bottom (response probability) row to accentuate the relatively subtle differences compared to the more pronounced contour differences in the upper (RT) row smaller than the equivalent physical difference in the, lower intensity, baseline condition leading to higher mean RT for the additive-boost condition in contradiction to our data (Fig. 3 column 2; Tables 1 and 2, Model 3).

To test the roles of the three sources of absolute value sensitivity within a differential relativity framework, we fit the full DDM model (Eq. 4) to the data from Exp. 1. The model captures all the data well both qualitatively and quantitatively (Tables 1 and 2, Model 4; Fig. 3). Interestingly, the best fit was achieved with a degenerate partial relativity coefficient $\alpha=1$ reducing to a model where the sensitivity to absolute values comes solely from the noise component $(\pi=0.69)$. Thus, within a DDM framework, our model fits provide support for the dominant role of multiplicative noise over partial modulation of stopping rule relativity.

\section{Dynamic relativity}

The LCA model (Eq. 5) captured all the empirical effects both qualitatively and quantitatively (Tables 1 and 2, Model 5; Fig. 3, rightmost column). Interestingly, the fit was achieved with $\pi<10^{-4}$ indicating no role for input dependent noise in contradiction to the DDM interpretation. 


\section{Input space analysis}

To better understand the dynamics and predictions of the different models, we performed a simulation-based computational investigation of input spaces. The best fitting average observer parameters for each model (as in Table S1) were used for these simulations. Using tractable transformations of physical stimulus values into model input values allowed us to derive model predictions for a continuum of possible stimulus value combinations, including the values used in the experiment (Fig. 3, bottom panel). The first observation that stands out is that models differ only minimally in their predictions for accuracy contours. Except for the purely relative DDM (model 4; Fig. 3, second column) where accuracies are approximately constant for equal differences (contours parallel to main diagonal), predictions of all models maintain approximately constant accuracies for constant input ratios (fanning out contours compared to the diagonal). The second observation is that RT dynamics vary qualitatively between different relative architectures. Purely fractional relativity leads to a fan like pattern that approximately maintains ratios (normalized race model; Table 1, model 1; Fig. 3, first column). Purely differential relativity predicts approximately parallel diagonal lines (DDM; Table 1, model 3; Fig. 3, second column) that maintain constant differences. Unlike purely relative models, RT dynamics for both the partially relative models (DDM with input dependent noise and the LCA model (Table 1, models 4 and 5; Fig. 3, two rightmost columns correspondingly) predict RT contour lines that are approximately parallel when the stimuli are clearly discriminable (far from the main diagonal) yet converge towards the main diagonal (rather than fanning out) for less discriminable stimuli. In other words, both partially relative models make the testable prediction that the size of the additive-boost RT speedup effect would be larger for less discriminable stimuli. The curvature of the heat contours suggests that there are quantitative differences between the two partial relativity architectures in the rate with which RT changes as a function of absolute input values. This provides an avenue for stronger future tests of the models by using several "boost" levels (e.g. baseline; baseline*5/4; baseline $* 3 / 2$; baseline +0.1 ; baseline $+\mathbf{0 . 2}$; bold represents current mean brightness values in Exp. 1 \& 2).

\section{Discussion}

In this study, we focused on assumptions regarding the relativity of information processing and its sensitivity to absolute values in order to learn about the mechanisms underlying perceptual decision making. Critically, our experimental results demonstrate a sensitivity of response latencies to both additive and multiplicative boosts in brightness intensity values, which was not predicted by purely relative models:
RTs speeded up with boosted brightness levels, even when the differences or the ratios were maintained. These effects constitute violations of value invariance predicted by purely relative models of decision making. The ratio and difference theoretical frameworks discussed in this study can be regarded as purely relative in that they forfeit all information about, and thus any sensitivity to, the absolute values representing the choice alternatives. Because successful performance in our discrimination task only requires attending to the relation of the two stimuli (i.e., which one is brighter relative to the other), this pure relativity assumption can be considered rational, at least in the sense that the absolute values are irrelevant to task performance.

Value sensitivity, as it was observed in this study, is reminiscent of Pieron's law whereby higher intensities lead to faster responses (Geisler 1989). However, previous demonstrations of Pieron's law have confounded higher intensity with higher signal to noise ratio (Van Maanen et al. 2012). This resulted in decisions being easier for the higher intensity conditions, as in the multiplicative boost condition in this study, thus allowing for a natural account within standard choice RT models. In the absence of specific constraints on the relationship between physical stimulus values and model inputs, higher signal to noise ratios are commonly associated with higher drift-rate differences (in DDM models) or higher drift-rate ratios (in normalization models). This, given a constant decision threshold, directly leads to faster RTs in most models. However, in our experiments, RTs were faster even in the additive boost condition where the signal to noise was not larger than the baseline condition. Therefore, our results constitute the first demonstration that Pieron's law holds even for conditions where higher intensity is associated with equal or lower signal to noise ratios compared with the lower intensity conditions. In addition, we replicate the results of Teodorescu and Usher (2013), demonstrating slower RTs for higher nontarget stimulus values, in contradiction to purely absolute models (i.e., independent race models), which predict the opposite pattern. Thus, our experimental framework provides a theoretically motivated benchmark manipulation for simultaneously testing multiple decision-making theories.

We contrasted models that varied with respect to the type of relative information processing (differential vs. fractional), the degree of relativity (on a purely absolute to purely relative continuum), lateral inhibition (an alternative neural mechanism for partially relative evidence integration) and the type of noise (constant or input dependent). The results of the model comparison rule out purely absolute (independent race) models, purely relative models of the differential type based on the integration of (psychophysically transformed) differences, and both pure and partially relative models of the fractional type based the integration of (psychophysically transformed) normalized inputs. Interestingly, fractional relativity captures the accuracy results quite well, and its failure applies 
almost exclusively to RTs, demonstrating the importance of fitting multiple dependent measures. In a recent study, normalization was used to explain relative effects of value manipulations on accuracy (Louie et al. 2011). The authors showed that increasing the value of a third, nonpreferred alternative changed the proportion of choices between the two preferred alternatives in favor of the second-best alternative. However, in our study the stronger nontarget value in the additive compared with the multiplicative boost produced slower RTs in addition to lower accuracy. While predicting the accuracy effect correctly, the normalization model did not produce an adequate RT slowdown. Thus, our results suggest that a reexamination of the Louie et al. dataset with respect to choice RTs might provide additional insights into the mechanisms underlying the decision process. Note that we rule out a normalization scheme that is plausible and prevalent in the literature. However, other normalization schemes can be potentially designed based on different principles other than ratios, which might be able to better account for our results. Nevertheless, our results demonstrate that any such scheme should incorporate some measure of absolute value sensitivity and cannot be purely relative.

Due to its purely relative nature, the DDM has been claimed to be incompatible with trial by trial sensitivity to absolute values as previously associated with models of social colony decision making (Pirrone et al. 2014) and now, as per our results, also with findings for human decision making. However, the neurally plausible assumption that processing noise is proportional to momentary input values, coupled with a differentially relative decision mechanism, resolves this challenge. The results of this study imply that the dependence of processing noise on input values is not by any measure just a technical assumption but one that has direct theoretical implication for the underlying psychological mechanism. The segregation of general processing noise into general and input dependent, multiplicative noise components also is consistent with the modeling work of Brunton et al. 2013 (cf. Lu and Dosher 2008). Interestingly, Brunton et al. found zero general processing noise and a major role for input dependent noise. However, their conclusion is contingent on using a differential relativity framework to fit accuracy data. In our computational study, the differential and dynamic relativity frameworks categorically disagreed regarding the role of multiplicative noise. Thus, beyond stressing the added value of fitting RTs, the current work suggests that conclusions about properties of internal processes such as the roles of general versus multiplicative noise can critically depend on the, often arbitrary, choice of modeling architecture.

Indeed, dynamic relativity also captured our results well, both quantitatively and qualitatively. The LCA model can be conceptualized intuitively as a dynamic amalgam of both absolute and differentially relative processing occurring at early and late stages respectively. Under some assumptions, the LCA is asymptotically equivalent to a DDM (Bogacz et al.
2006; Bogacz et al. 2007; Marshall et al. 2009), but with lower decision thresholds for conditions involving higher input values. Interestingly, the two models provide two incompatible accounts for our data. Specifically, the DDM used a purely relative stopping rule such that input dependent multiplicative noise was solely responsible for producing intensity related RT-speedup effects. Conversely, multiplicative noise played a detrimental role in the LCA, where speedups were uniquely produced by value sensitivity during the initial stages of the gradual transition from purely absolute to increasingly relative processing as a result of lateral inhibition. Conceptually, the account provided by the LCA can be considered intrinsic to the architecture of the decision mechanism as the RT speedup is produced by sensitivity to absolute values during the early stages of evidence accumulation. On the other hand, the account provided by the DDM can be considered extrinsic, because it is based on the scaling properties of neural noise, which are independent from the decision mechanism. Indeed, both accounts could be the end products of evolutionary pressures. However, while the former seems to suggest an evolved value sensitive design feature, the latter is more compatible with a mechanical limitation of information processing, not eliminated through natural selection due either to a lack of a better alternative or to the benefits of value sensitivity (expedited decisions for higher values) overweighing its disadvantages (lower accuracy for equally discriminable higher values).

More generally, this study provides an observation of what could be considered an involuntary, value dependent, bottom up, speed-accuracy tradeoff (SAT; Heitz 2014; Pirrone et al. 2014). We found that for equal or lower stimulus discriminability, high-intensity stimuli bias decision making towards faster decisions at the expense of higher error-rates. Deliberate, top-down SATs have been the subject of extensive investigation in psychology, traditionally studied by manipulating either the subject's goals (e.g. respond fast vs. respond accurately) or by controlling the subjects RT directly via response cues (Heitz 2014). However, such voluntary criterion modulations are relatively slow, effortful, and require executive control based on intricate understanding of the context, making them inefficient for dealing with trial by trial variations in decision values.

Although the sensitivity to absolute values appears to violate "rationality" in the narrow sense, it is possible that it has an adaptive value in a broader ecological sense, which includes typical tasks and environmental contingencies. This relates to a different kind of tradeoff, the speed-value tradeoff, which has been recently suggested as more appropriate outside the laboratory (Pirrone et al. 2014). Most decisions in naturalistic environments involve value, rather than accuracy based rewards, whereby the agent is rewarded in proportion to the value of the chosen alternative and not with constant rewards for objectively correct ("best" alternative) responses. In 
such environments, there are several reasons why decisions between high-value alternatives may warrant expedited responses (i.e., speed-value tradeoff). First, the set of available alternatives is not always static, which often is the case in the laboratory. Thus, taking a long time to choose might allow for additional alternatives to present themselves (Pais et al. 2013; Pirrone et al. 2014). If the values of the alternatives considered are low, a new alternative is more likely to be better than the existing ones. In contrast, in a situation where the existing alternatives are already high valued, new alternatives are unlikely to provide benefits but existing (high-valued) alternatives could expire. Second, unlike monetary rewards, which can be infinitely hoarded, many natural resources relevant to survival and reproduction have a short lifespan. Take for example a hungry agent deliberating between two unoccupied, fruit-laden patches of berries on opposite sides of a valley. When such perishable resources are abundant (high value of redness), it is unlikely one could take advantage or consume all of the reward, making deliberation over difference in the absolute quantities less relevant and favoring a quick decision over a slow but objectively "correct" one. In addition, intense perceptual values can indicate abundance but are also more salient and thus more likely to attract competition. Consequently, dallying for too long in deciding could result in these alternatives being occupied by someone else and leading to loss of resources or potential conflict. Alternatively, when dealing with negative rewards that are to be avoided, high-stimulus intensities also could serve as a cue for danger (e.g., the fast motion of an incoming projectile or predator; the loud noise of a stampeding herd or rock avalanche, etc.). The potentially high cost of not reacting in time to such high intensity stimuli, could again support quick and frugal reactions over making an "accurate" response too late.

Therefore, a mechanism that allows speeded reactions to high intensity situations in a bottom-up fashion, might be meta-optimal in the sense that, beyond providing satisfying decision quality in most everyday situations, it also captures the merit of expedited decisions under certain unexpected situations characterized by high intensity stimuli. Our study provides evidence for violations of invariance to absolute values and suggests that partially relative information processing is both necessary and sufficient for producing the observed value sensitivity.

Recent studies on decentralized decision making in biological systems, such as house hunting bees, revealed parallels between neural mechanism responsible for decision making in the human brain and collective decision making in social colonies (Seeley et al. 2012). Indeed, insights from modeling human decision making with lateral inhibition as in the LCA model have proven useful in modeling bee colony behavior (Marshall et al. 2009; Pirrone et al. 2014). These parallels suggest similar evolutionary pressures across species whereby retaining sensitivity to absolute values in addition to relative ones might hold adaptive advantages (Pais et al. 2013). The mechanism underlying value sensitivity in both humans and social colonies could be the result of an evolved advantage mediated by lateral inhibition or an accidentally beneficial side effect of mechanical limitations on the variability of information processing. Either way, distinguishing between these two hybrid theoretical frameworks would require an integrated approach. To this end, future investigations, in which the type of noise and the nonlinearity are measured via more complex psychophysical procedures (Brunton et al. 2013; Lu and Dosher 2008) could be used in conjunction with dedicated intensity manipulations of the type presented in this study.

Author note 1) A. T. was funded by the Fulbright Scholar Program. 2) M.U. is funded by the Israeli Science Foundation (grant: 743/12) and by the German Israeli Foundation (grant, 1130-158.4/2010).

\section{References}

Akaike, H. (1974). A new look at the statistical model identification. IEEE Transactions on Automatic Control, 19. doi:10.1109/TAC. 1974.1100705

Basten, U., Biele, G., Heekeren, H. R., \& Fiebach, C. J. (2010). How the brain integrates costs and benefits during decision making. Proceedings of the National Academy of Sciences of the United States of America, 107(50), 21767-21772. doi:10. 1073/pnas.0908104107

Bogacz, R., Brown, E., Moehlis, J., Holmes, P., \& Cohen, J. D. (2006). The physics of optimal decision making: A formal analysis of models of performance in two-alternative forced-choice tasks. Psychological Review, 113(4), 700-765. doi:10.1037/0033-295X. 113.4.700

Bogacz, R., Brown, E., Moehlis, J., Holmes, P., \& Cohen, J. D. (2006). The physics of optimal decision making: A formal analysis of models of performance in two-alternative forced-choice tasks. Psychological Review, 113(4), 700-765. doi:10.1037/0033-295X. 113.4.700

Bogacz, R., Usher, M., Zhang, J., \& McClelland, J. L. (2007). Extending a biologically inspired model of choice: Multi-alternatives, nonlinearity and value-based multidimensional choice. Philosophical Transactions of the Royal Society of London. Series B, Biological Sciences, 362(1485), 1655-1670. doi:10.1098/rstb.2007.2059

Brown, S. D., \& Heathcote, A. (2008). The simplest complete model of choice response time: Linear ballistic accumulation. Cognitive Psychology, 57(3), 153-178. doi:10.1016/j.cogpsych.2007.12.002

Brunton, B. W., Botvinick, M. M., \& Brody, C. D. (2013). Rats and humans can optimally accumulate evidence for decision-making. Science (New York, N.Y.), 340(6128), 95-98. doi:10.1126/science. 1233912

Carandini, M., \& Heeger, D. J. (2012). Normalization as a canonical neural computation. Nature Reviews. Neuroscience, 13(1), 51-62. doi:10.1038/nrn3136

Cousineau, D. (2005). Confidence intervals in within-subject designs: A simpler solution to Loftus and Masson's method. Tutorials in Quantitative Methods for Psychology, 1(1), 42-45. Retrieved from http://www.tqmp.org/Content/vol01-1/p042/p042.pdf

Drugowitsch, J., Moreno-Bote, R., Churchland, A. K., Shadlen, M. N., \& Pouget, A. (2012). The cost of accumulating evidence in perceptual decision making. The Journal of Neuroscience: The Official 
Journal of the Society for Neuroscience, 32(11), 3612-3628. doi:10. 1523/JNEUROSCI.4010-11.2012

Geisler, W.S. (1989). Sequential ideal-observer analysis of visual discriminations. Psychological Review, 96(2), 267-314. Retrieved from http://www.ncbi.nlm.nih.gov/pubmed/2652171

Heathcote, A. (1998). Neuromorphic models of response time. Australian Journal of Psychology, 50(3), 157-164. doi:10.1080/ 00049539808258792

Heathcote, A., \& Brown, S. (2004). Reply to Speckman and Rouder: A theoretical basis for QML. Psychonomic Bulletin \& Review, 11(3), 577-578. doi:10.3758/BF03196614

Heathcote, A., Brown, S., \& Cousineau, D. (2004). QMPE: Estimating lognormal, Wald, and Weibull RT distributions with a parameterdependent lower bound. Behavior Research Methods, Instruments, \& Computers : A Journal of the Psychonomic Society, Inc, 36(2), 277-290. doi:10.3758/BF03195574

Heitz, R.P. (2014). The speed-accuracy tradeoff: History, physiology, methodology, and behavior. Frontiers in Neuroscience, 8(June), 150. doi:10.3389/fnins.2014.00150

Jones, M., \& Dzhafarov, E. N. (2013). Unfalsifiability and mutual translatability of major modeling schemes for choice reaction time. Psychological Review. doi:10.1037/a0034190

Louie, K., Grattan, L. E., \& Glimcher, P. W. (2011). Reward valuebased gain control: Divisive normalization in parietal cortex. The Journal of Neuroscience: The Official Journal of the Society for Neuroscience, 31(29), 10627-10639. doi:10.1523/JNEUROSCI. 1237-11.2011

Louie, K., Grattan, L. E., \& Glimcher, P. W. (2011). Reward value-based gain control: Divisive normalization in parietal cortex. The Journal of Neuroscience : The Official Journal of the Society for Neuroscience, 31(29), 10627-10639. doi:10.1523/JNEUROSCI. 1237-11.2011

Louie, K., Khaw, M. W., \& Glimcher, P. W. (2013). Normalization is a general neural mechanism for context-dependent decision making. Proceedings of the National Academy of Sciences of the United States of America, 110(15), 6139-6144. doi:10.1073/pnas. 1217854110

Lu, Z.-L., \& Dosher, B. A. (2008). Characterizing observers using external noise and observer models: Assessing internal representations with external noise. Psychological Review, 115(1), 44-82. doi:10. 1037/0033-295X.115.1.44

Marshall, J. A. R., Bogacz, R., Dornhaus, A., Planqué, R., Kovacs, T., \& Franks, N. R. (2009). On optimal decision-making in brains and social insect colonies. Journal of the Royal Society, Interface / The Royal Society, 6(40), 1065-1074. doi:10.1098/rsif.2008.0511

Meyer, D.E., Irwin, D.E., Osman, A.M., \& Kounios, J. (1988). The dynamics of cognition and action: Mental processes inferred from speed-accuracy decomposition. Psychological Review, 95(2), 183-237. Retrieved from http:/www.ncbi.nlm.nih.gov/ pubmed/3375399

Moran, R. (2014). Optimal decision making in heterogeneous and biased environments. Psychonomic Bulletin \& Review. doi:10.3758/ s13423-014-0669-3

Moran, R., Zehetleitner, M., Müller, H. J., \& Usher, M. (2013). Competitive guided search : Meeting the challenge of benchmark RT distributions. Journal of Vision, 13(8), 1-31. doi:10.1167/13.8.24

Moreno-Bote, R. (2010). Decision confidence and uncertainty in diffusion models with partially correlated neuronal integrators. Neural Computation, 22(7), 1786-1811. doi:10.1162/neco.2010.12-08-930

Nelder, J. A., \& Mead, R. (1965). A simplex method for function minimization. Computer Journal, 7(4), 308-313. doi:10.1093/comjn1/7. 4.308

Ossmy, O., Moran, R., Pfeffer, T., Tsetsos, K., Usher, M., \& Donner, T. H. (2013). The timescale of perceptual evidence integration can be adapted to the environment. Current Biology, 23, 981-986. doi:10. 1016/j.cub.2013.04.039
Pais, D., Hogan, P.M., Schlegel, T., Franks, N.R., Leonard, N.E., \& Marshall, J.A.R. (2013). A mechanism for value-sensitive decisionmaking. PloS One, 8(9), e73216. doi:10.1371/journal.pone.0073216

Pirrone, A., Stafford, T., \& Marshall, J.A.R. (2014). When natural selection should optimize speed-accuracy trade-offs. Frontiers in Neuroscience, 8(April), 73. doi:10.3389/fnins.2014.00073

Raftery, A.E. (1995). Bayesian model selection in social research. Sociological Methodology, 25, 111-163. Retrieved from https://www.stat.washington.edu/raftery/Research/PDF/ socmeth1995.pdf

Ratcliff, R. (1978). A theory of memory retrieval. Psychological Review 85(2), 59-108. doi:10.1037/0033-295X.85.2.59

Ratcliff, R., \& McKoon, G. (2008). The diffusion decision model: Theory and data for two-choice decision tasks. Neural Computation, 20(4), 873-922. doi:10.1162/neco.2008.12-06-420

Ratcliff, R., \& Rouder, J. N. (1998). Modeling response times for twochoice decisions. Psychological Science, 9(5), 347-356. doi:10. 1111/1467-9280.00067

Ratcliff, R., \& Smith, P. L. (2004). A comparison of sequential sampling models for two-choice reaction time. Psychological Review, 111(2), 333-367. doi:10.1037/0033-295X.111.2.333

Roe, R. M., Busemeyer, J. R., \& Townsend, J. T. (2001). Multialternative decision field theory: A dynamic connections $\mathrm{t}$ model of decision making. Psychological Review, 108(2), 370-392. doi:10.1037// 0033-295X.108.2.370

Schwarz, G. (1978). Estimating the dimension of a model. The annals of statistics, 6(2), 461-464

Seeley, T.D., Visscher, P.K., Schlegel, T., Hogan, P.M., Franks, N.R., \& Marshall, J.A.R. (2012). Stop signals provide cross inhibition in collective decision-making by honeybee swarms. Science, 335(6064), 108-111.

Teodorescu, A. R., \& Usher, M. (2013). Disentangling decision models: From independence to competition. Psychological Review, 120(1), 1-38. doi:10.1037/a0030776

Towal, R.B., Mormann, M., \& Koch, C. (2013). Simultaneous modeling of visual saliency and value computation improves predictions of economic choice. Proceedings of the National Academy of Sciences of the United States of America, 110(40), E3858-67. doi:10.1073/ pnas. 1304429110

Trueblood, J. S., Brown, S. D., Heathcote, A., \& Busemeyer, J. R. (2013). Not just for consumers: Context effects are fundamental to decision making. Psychological Science, 24(6), 901-908. doi:10.1177/ 0956797612464241

Tsetsos, K., Chater, N., \& Usher, M. (2012). Salience driven value integration explains decision biases and preference reversal. Proceedings of the National Academy of Sciences of the United States of America, 109(24), 9659-9664. doi:10.1073/pnas.1119569109

Tversky, A., \& Simonson, I. (1993). Context-dependent preferences. Management Science, 39(10), 1179-1189. doi:10.1287/mnsc.39. 10.1179

Usher, M., Olami, Z., \& McClelland, J. L. (2002). Hick's law in a stochastic race model with speed-accuracy tradeoff. Journal of Mathematical Psychology, 46(6), 704-715. doi:10.1006/jmps. 2002.1420

Usher, M., \& McClelland, J. L. (2001). The time course of perceptual choice: The leaky, competing accumulator model. Psychological Review, 108(3), 550-592. doi:10.1037//0033-295X.108.3.550

Usher, M., \& McClelland, J. L. (2004). Loss aversion and inhibition in dynamical models of multialternative choice. Psychological Review, 111(3), 757-769. doi:10.1037/0033-295X.111.3.757

Van Maanen, L., Grasman, R. P. P. P., Forstmann, B. U., \& Wagenmakers, E.-J. (2012). Piéron's law and optimal behavior in perceptual decision-making. Frontiers in Neuroscience, 5, 143. doi:10.3389/fnins. 2011.00143 
Van Zandt, T., Colonius, H., \& Proctor, R. W. (2000). A comparison of two response time models applied to perceptual matching. Psychonomic Bulletin \& Review, 7, 208-256.

Vickers, D. (1970). Evidence for an accumulator model of psychophysical discrimination. Ergonomics, 13, 37-58. doi:10.1080/ 00140137008931117
Wald, A., \& Wolfowitz, J. (1948). Optimum character of the sequential probability ratio test. The Annals of Mathematical Statistics, 19(3), 326-339. doi:10.1214/aoms/1177730197

Zylberberg, A., Barttfeld, P., \& Sigman, M. (2012). The construction of confidence in a perceptual decision. Frontiers in Integrative Neuroscience, 6(September), 79. doi:10.3389/fnint.2012.00079 